\title{
SIU-ICUD consultation on bladder cancer 2018
}

\author{
Peter Black $^{1} \cdot$ Paolo Gontero $^{1}$
}

Published online: 21 January 2019

c) Springer-Verlag GmbH Germany, part of Springer Nature 2019

With nearly 120,000 new cases and 20,000 deaths in Europe in 2012 and more than 60,000 new cases and 12,000 deaths in the USA alone in 2018, the burden of bladder cancer in health care continues to increase. The incidence of bladder cancer peaks in the 7th decade [1], which means that an enormous disease burden is expected as the global population ages.

Taking these figures into account, in 2017 the Société Internationale d'Urologie (SIU) teamed up with the International Consultation on Urological Diseases (ICUD) to convene a Consultation on Bladder Cancer. This was warranted due to the rapid advances in the field of bladder cancer care and research. Many key opinion leaders from around the world led nine committees, with each committee dedicated to one important component of clinical care and research. A meeting during the 2017 SIU Congress in Lisbon, Portugal provided an open forum for debate and discussion of the most recent advances and the most controversial issues in the field, and final reports from each committee incorporated feedback from this session. The reports from each of the nine committees have been summarized in this special SIU issue of the Journal.

The SIU-ICUD consultation on bladder cancer has tackled some potentially contentious issues and addressed all the recent advances in the field. In the context of localized muscle-invasive disease, for example, the recent randomized trials comparing robotic and open radical cystectomy have been reviewed, and the impact of trimodal therapy as an alternative to radical cystectomy has been highlighted by Stenzl et al. (current issue). The section on urinary diversion has also critically apprised the issue of open versus robotic surgery, but in the context of intracorporeal urinary diversion after radical cystectomy (Rodriguez-Faba et al. current issue).

Paolo Gontero

paolo.gontero@unito.it

1 Department of Surgical Sciences, Molinette Hospital, University of Torino School of Medicine, C.so Bramante 88/90, 10126 Turin, Italy
One of the biggest advances in the management of bladder cancer has been the advent of systemic immunotherapy for patients with metastatic bladder cancer. This became a key part of the section on systemic therapy (Steingerg et al. current issue) and was also relevant in the section on molecular markers (Shariat et al. current issue) and basic science (McConkey et al. current issue).

Precision oncology is finally making an impact on bladder cancer as it has in most other cancers. We have witnessed a notable increase in preclinical and early translational research that is greatly improving our understanding of the molecular landscape of bladder cancer. In this context, two key chapters of the Consultation were devoted to address molecular markers and basic science. The update on molecular markers provides a comprehensive overview of the currently available evidence on serum, urine and tissue markers in bladder cancer. Although these currently have limited clinical applicability, several new markers are likely to replace traditional diagnostic and surveillance strategies in the near future (Soria et al. current issue).

For the first time, the SIU-ICUD Consultation includes a section on the basic science of bladder cancer (McConkey et al. current issue). This section has developed into a critical component of the Consultation that does not exist in this form in any similar document. A team of highly accomplished scientists from around the globe has assembled a comprehensive, but concise overview of a broad spectrum of topics, from molecular subtypes and metabolomics, to animal models and bladder cancer stem cells. This section should become essential reading for all urologic trainees in the clinic and the laboratory, as well as those interested in catching up with all recent advances in this field.

The section on pathology contains some exciting features, including especially current criteria for morphologic classification of variant histologic subtypes and new concepts on immunohistochemistry for disease classification (Comperat et al. current issue). New elements of staging and grading have been incorporated, as well as content from the 2017 International Collaboration on Cancer Reporting as it pertains to bladder cancer. A separate chapter on non-urothelial 
histology delves especially into the clinical ramifications of the different types of bladder cancer (Konety et al. current issue).

Equally important are the sections on epidemiology, prevention, screening, diagnosis, and evaluation (Kamat et al. current issue), and on the management of non-muscle-invasive bladder cancer (Kassouf et al. current issue).

The SIU-ICUD Joint Consultation on Bladder Cancer represents a urologic tour de force that provides a critical resource and an invaluable international reference on bladder cancer for all providers treating and studying this disease.
The summaries of each chapter of the Consultation published in this edition of the Journal are important in disseminating this work to a broad international audience.

\section{Reference}

1. Siegel RL, Jemal A, Wender RC et al (2018) An assessment of progress in cancer control. CA Cancer J Clin 68(5):329-339 